\title{
Characterization of Hardware Impairments in Multiple Antenna Systems for DoA Estimation
}

\author{
Daniele Inserra and Andrea M. Tonello \\ Dipartimento di Ingegneria Elettrica, Gestionale e Meccanica, Università degli Studi di Udine, \\ Via delle Scienze 208, 33100 Udine, Italy \\ Correspondence should be addressed to Andrea M. Tonello, tonello@uniud.it
}

Received 25 July 2011; Accepted 12 September 2011

Academic Editor: Nikos Sagias

Copyright ( 2011 D. Inserra and A. M. Tonello. This is an open access article distributed under the Creative Commons Attribution License, which permits unrestricted use, distribution, and reproduction in any medium, provided the original work is properly cited.

\begin{abstract}
We consider the effects introduced by hardware impairments on the estimation of the direction of arrival (DoA) deploying a multiple antenna radio system. We derive a system model from experimental measurements of a state-of-the-art hardware test bed based on an RF direct-conversion architecture. The system model includes DC offsets, carrier frequency and phase offsets, and the phase noise. Then, we propose a simple digital compensation algorithm of the impairments for bidimensional DoA estimation (azimuth and elevation) with a 3D orthogonal linear array. The robustness of the algorithm in terms of minimum-mean-squared error as a function of the hardware impairments is evaluated and compared with that achieved using the root-MUSIC algorithm.
\end{abstract}

\section{Introduction}

The estimation of the direction of arrival (DoA) in wireless radio systems with the use of antenna arrays has attracted considerable interest. The DoA estimation can be exploited for positioning and tracking of wireless nodes, for navigation, to support context aware communication services in cellular, vehicular, and wireless sensor networks. The fundamental aspects of plane waves propagation and the properties of planar arrays that allow DoA estimation were discussed in the early work [1]. It was shown that the delays of the signals in individual array elements are a function of the DoA. Over the years several improved approaches have been proposed as the algorithms based on the maximum likelihood paradigm [2], an approach that is however computationally intensive, and the so-called subspace-based methods $[3,4]$ that are particularly suited for the DoA estimation of multiple emitters.

An important aspect in DoA estimation is the presence of hardware imperfections. The array uncalibration is among the most studied problems [5-8]. It comprises the gain and phase mismatch among the array elements (sensors) and the unknown position of the sensors. If a reference signal is available, precalibration of the array is possible as proposed in [8]. Mutual coupling among the sensors may also be present, although it has been shown that it has lower detrimental effect than the phase mismatch $[8,9]$. The accurate design of the array can significantly mitigate the uncertainty of the sensor position and the mutual coupling among them.

However, there are other impairments introduced by both the RF and acquisition (baseband) hardware stages that cannot be neglected. In particular, carrier frequency and phase offsets can have a detrimental effect on the DoA estimation algorithm if they are not compensated [9]. Furthermore, the presence of DC offsets and phase noise can also limit the performance of the estimator. We have observed the presence of DC offsets, phase noise, phase, and carrier frequency offsets, through the experimental development of a hardware test bed that deploys a stateof-the-art multichannel direct-conversion receiver followed by an acquisition board based on the Lyrtech platform [10]. Although these hardware impairments are usually considered in the context of data transmission systems, for example, in [11-13], they are often neglected in the context of DoA estimation with antenna arrays.

In this paper, we consider the DoA estimation problem in the presence of hardware impairments. We assume a 
single transmitting node in a plane waves line-of-sight (LOS) propagation scenario. Multiple-node transmission can be accomplished by time or frequency multiplexing the node transmissions so that the single source model applies. The contribution of this paper is threefold:

(a) we report the results of experimental measurements and we derive a system model for a typical directconversion hardware architecture that is affected by DC offsets, carrier frequency offsets, phase offsets, and phase noise;

(b) we propose a simple compensation algorithm that copes with such impairments and allows 2D DoA estimation using L-shaped arrays;

(c) we report several performance results obtained via simulations and compare them with those attained with a $2 \mathrm{D}$ root-MUSIC algorithm [14] that we have appropriately adapted to be used in our specific context.

Some preliminary results were described in $[15,16]$ where, however, we did not consider the effect of phase noise, we used a different and more heuristic DC offset model, and we deployed a different array calibration procedure yielding a different DoA algorithm.

The paper is organized as follows. In Section 2, we describe the measurement results of the hardware test bed from which we have derived the statistical model of the impairments. In Section 3, the system model for DoA estimation with these imperfections is presented. The proposed 2D DoA estimation algorithm is described in Section 4. We study the mean-square-error performance of the estimator in Section 5. The results obtained are compared with those attainable with the root-MUSIC. Finally, the conclusions follow.

\section{Hardware Test Bed Description and Statistical Model Derivation}

In order to characterize and model the hardware impairments we have carried out a measurement activity on a stateof-the-art test bed, based on a Lyrtech platform [10]. The test bed deploys four RF direct-conversion receivers (that can operate either at $2.4 \mathrm{GHz}$ or at $5.8 \mathrm{GHz}$ ), followed by an eight channel acquisition board with FPGA. The analog-to-digital converters have 14-bit resolution with sampling rate up to $100 \mathrm{MHz}$. This allows to process four I\&Q channels, that is, to deploy an array with four elements.

In Figure 1, we depict the main blocks of the receiver architecture of our test bed for an antenna path. As we can observe, the signal captured by the antenna is filtered with a band select filter (BSF), amplified with a low-noise amplifier (LNA), and downconverted by two mixers fed by two $90^{\circ}$ delayed carriers. The downconverted signals are filtered with a channel select filter that attenuates the interferers and DC offset. Finally, we have a variable gain amplifier (VGA) and an antialiasing filter (AAF) that renders the signals suitable for the acquisition through the ADCs. The carrier frequency is generated by a phase-locked loop (PLL) from a lowfrequency reference oscillator shared by all the receivers.

The direct-conversion architecture is attractive due to its simplicity and the reduced costs, but it is well known that it has some drawbacks, among which the presence of DC offsets and the $I$ and $Q$ mismatch [17]. Furthermore, the finite precision and nonideality of the local oscillator introduces a frequency offset between the transmitter and each receiver carrier as well as phase noise. The measurements have revealed that the major impairments are the $\mathrm{DC}$ offset, the carrier frequency offsets, the phase noise, and the different, phases among the receiver signals that are introduced by both the time delays of unequal cable connections between the array elements, and not cophased local oscillators. We have found that the $I$ and $Q$ gain mismatch and the sampling time offsets introduced by the ADCs are of negligible entity in our application context.

In the following sections, we report the results of the measurements from which we derive and propose a model for the major impairments. The model allows the derivation of digital compensation algorithms and analysis of performance.

2.1. DC Offset. Both the RF downconversion stage and the acquisition board imperfections contribute to the DC offset. The RF stage contribution is due to signal leaking and self-mixing and to transistor mismatches in the signal path $[13,17]$. The ADC internal circuit imperfections give also a contribution to the DC offset.

In order to characterize the DC offset we have performed several acquisitions for all eight digital channels of our test bed. We have found that in our specific case the DC offset is practically constant although it is different among the channels. As an example, the measured DC offsets for the eight channels when we inject a $50 \mathrm{mV}$ sinusoidal tone are equal to $-6.0 \mathrm{mV},-22.5 \mathrm{mV},-10.8 \mathrm{mV},-24.9 \mathrm{mV}, 2.5 \mathrm{mV}$, $-13.2 \mathrm{mV}, 4.0 \mathrm{mV}$, and $-16.3 \mathrm{mV}$.

To study the statistics of the DC offset, we would need a wide set of receivers, which is clearly not doable in our case. We infer a Gaussian distribution from the observation of the physical phenomena that determine it in a large number of hardware realizations. The Gaussian model was also proposed in [13]. Furthermore, we assume the DC offsets to be statistically independent among the receiver channels, with zero mean and a given standard deviation. For example, the standard deviation of the measured DC offsets among the channels is equal to $10.6 \mathrm{mV}$. While the DC offset is independent of the input signal frequency, it depends on the level of the input signal. The relation has been obtained via measurements and it is reported in Figure 2 in terms of the ratio between the signal power $S^{2}$ at the output of the RF front-end and the DC offset power $N_{1}$ :

$$
\operatorname{SDR}=\frac{S^{2}}{N_{1}} .
$$

The figure shows that the test bed exhibits an SDR ranging from $16 \mathrm{~dB}$ to $26 \mathrm{~dB}$ with input signal amplitudes from $50 \mathrm{mV}$ to $100 \mathrm{mV}$. 


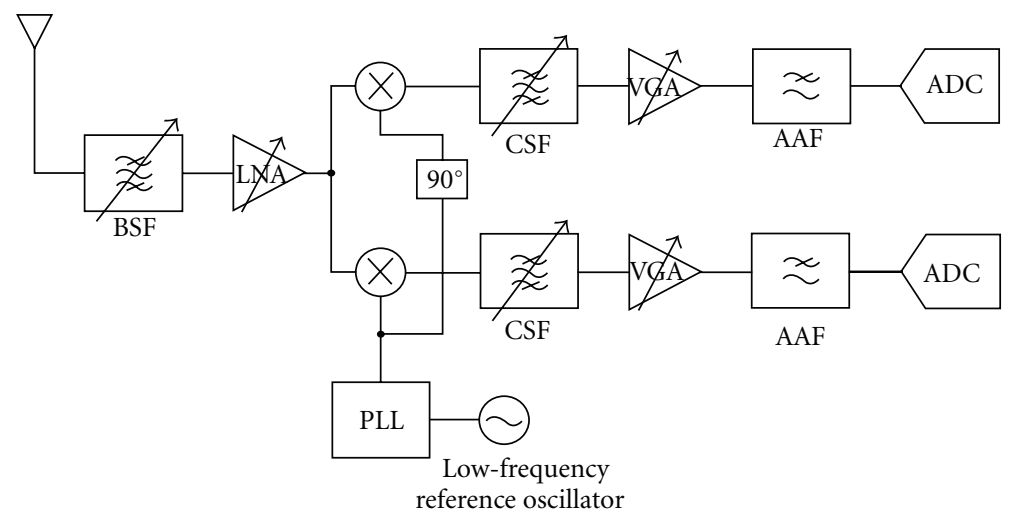

FIGURE 1: Receiver architecture for each channel (antenna) of our testbed.

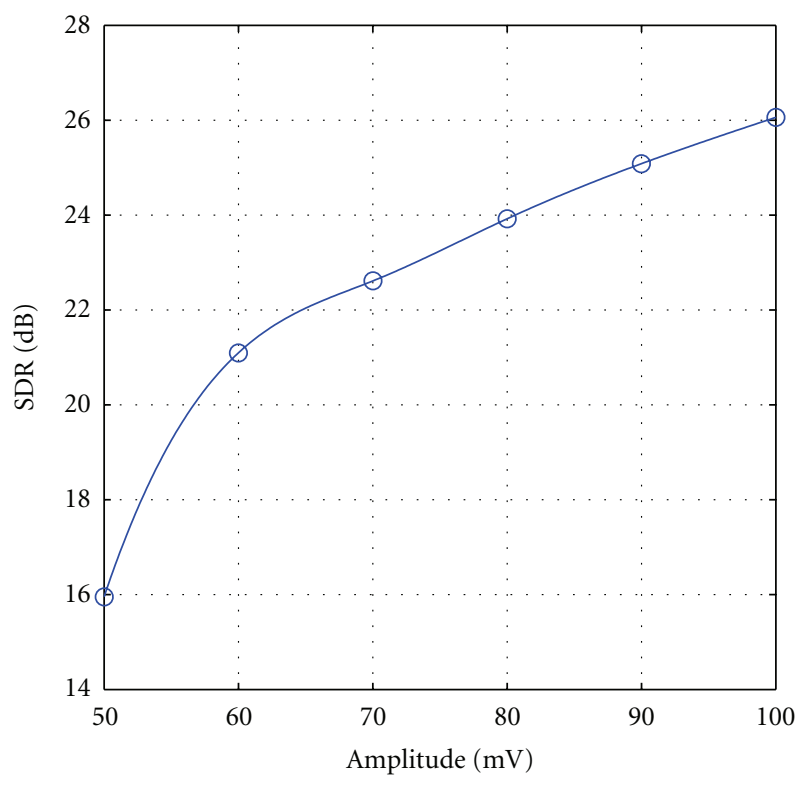

FIGURE 2: SDR as a function of the signal amplitude.

2.2. Phase Noise and Carrier Frequency Offset. The characterization of the phase noise and carrier frequency offset has been experimentally done by feeding each RF receiver with a single tone signal at the nominal carrier frequency. Then, the normalized received baseband output signal for the $i$ th channel sampled at rate $1 / T$ can be written as

$$
x^{(i)}(n T)=e^{j\left(2 \pi \Delta f^{(i)} n T+\varphi^{(i)}(n T)+\Phi^{(i)}\right)}+w^{(i)}(n T),
$$

where $\Delta f^{(i)}$ represents the frequency offset between the transmitted signal carrier and the carrier of the $i$ th receiver, while $\Phi^{(i)}$ is a constant phase offset. It should be noted that also phase noise can be present. It is herein denoted by $\varphi^{(i)}(n T)$ and it represents a time variant random component of the phase. Finally, $w^{(i)}(n T)$ is the background noise.

In Figure 3(a), we report the power spectrum, in $\mathrm{dBc} / \mathrm{Hz}$, of the phase noise $\varphi^{(i)}(n T)$ computed from the acquired samples of a given channel using the periodogram. For comparison, we also report the result of the measurement performed with a spectrum analyzer of the baseband signal at the output of the RF downconverter. As the figure shows, there is good consistency between the two power spectra.

In order to propose an analytical model for the phase noise, we use the parametric approach in [18] where the phase noise power spectrum is modeled as

$$
R_{\phi}(f)=10^{-c}+ \begin{cases}10^{-a}, & |f| \leq f_{1} \\ 10^{-\left|f-f_{1}\right|\left(b /\left(f_{2}-f_{1}\right)\right)-a}, & |f| \geq f_{1}\end{cases}
$$

for certain parameters $a, b, c, f_{1}$, and $f_{2}$. In particular, we have found that $a=5.3, b=3, c=7.8, f_{1}=$ $20 \mathrm{kHz}$, and $f_{2}=500 \mathrm{kHz}$ provide a good fit between the analytical expression and the measured results. Interestingly, as Figure 3(b) shows, the phase noise in our test bed is significantly higher than the more benign phase noise model used in [18] where the parameters are $a=6.5, b=4$, $c=10.5, f_{1}=1 \mathrm{kHz}$, and $f_{2}=10 \mathrm{kHz}$.

We have also statistically characterized the observed phase noise process. As Figure 4(a) shows, for a given receiver channel, it has a normal distribution with zero mean and standard deviation $1.57^{\circ}$. Similar values have been obtained for the other three channels. It has also been verified that the phase noise processes of distinct receivers are uncorrelated. Furthermore, the phase noise is slowly time variant. In fact, as shown in Figure 4(b), the correlation is larger than 0.55 after 25 samples (corresponding to $0.5 \mu$ s with sampling period $T=20 \mathrm{~ns}$ ).

Now, the phase offset $\Phi^{(i)}$ in (2) includes the phase offsets among the transmit-receive RF oscillators, the contribution due to delays of the signals that propagate through different length cables connecting the array elements with the RF board, and the phase difference among not cophased receiver local oscillators. These phase offsets can be assumed uniformly distributed in the range $[0,2 \pi]$ and constant during the observation window. In fact, the different PLLs control loops could generate a slow time variation of the phase differences among the channels which can be neglected since the considered observation window is sufficiently short.

Finally, the measurement of the carrier frequency offsets has shown that it has small differences among the four receivers with values $\Delta f^{(1)}=15.63 \mathrm{kHz}, \Delta f^{(2)}=15.44 \mathrm{kHz}$, 


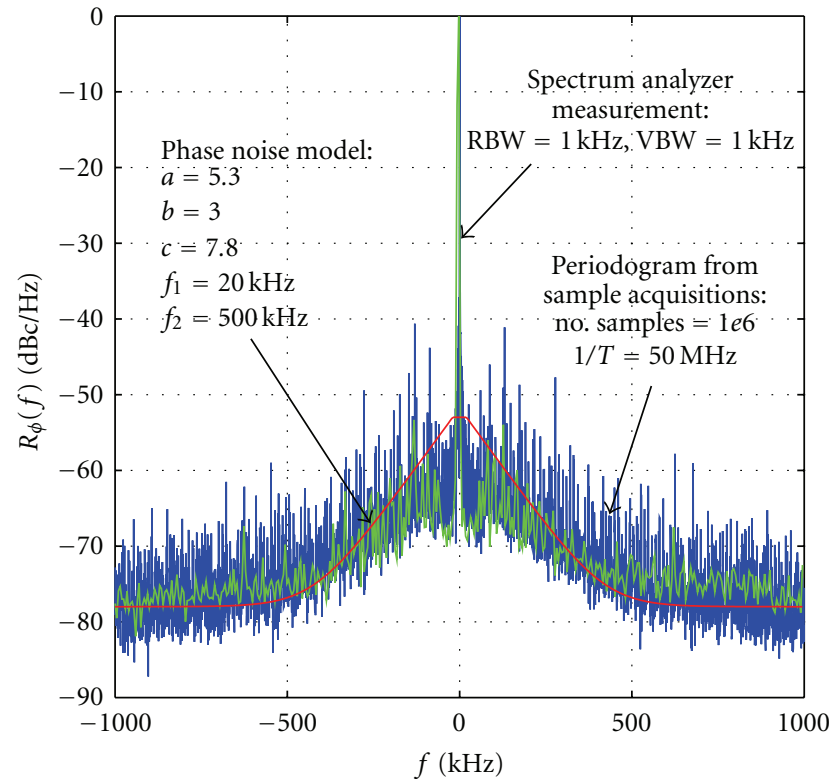

(a) Measured phase noise spectrum

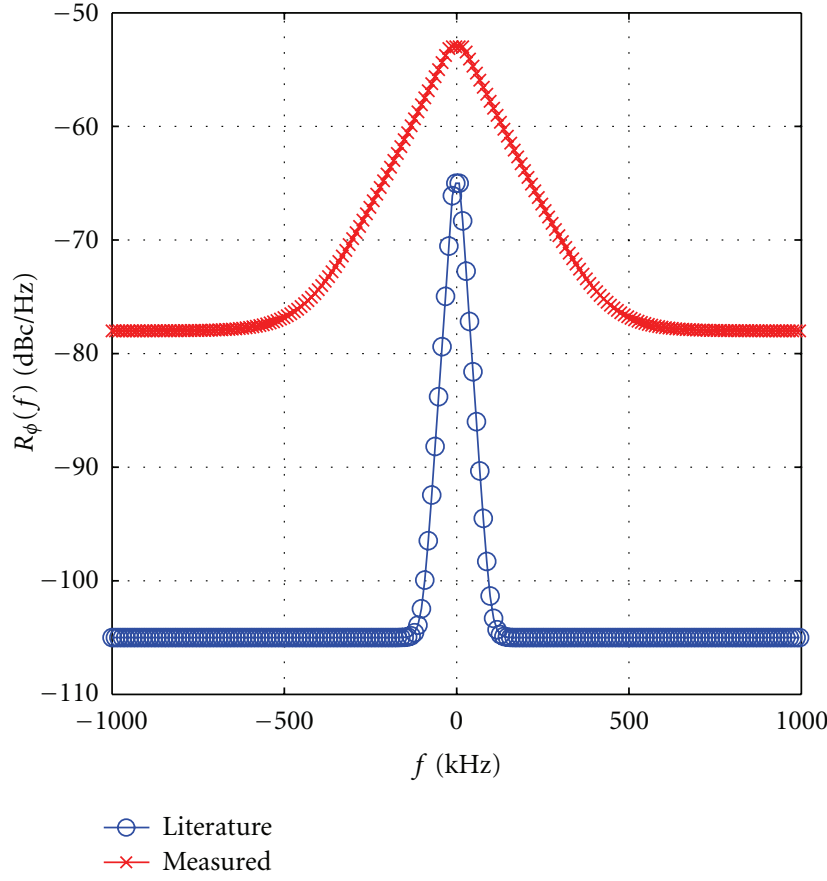

(b) Phase noise spectrum models

Figure 3: Phase noise spectrum measurements and models.

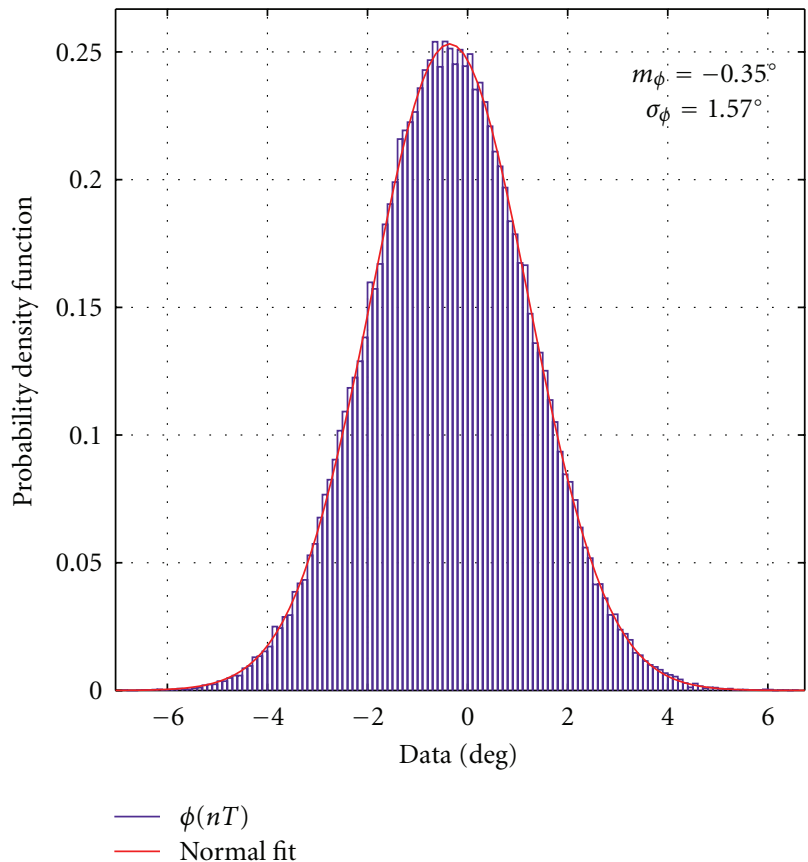

(a) Probability density function of the measured phase noise

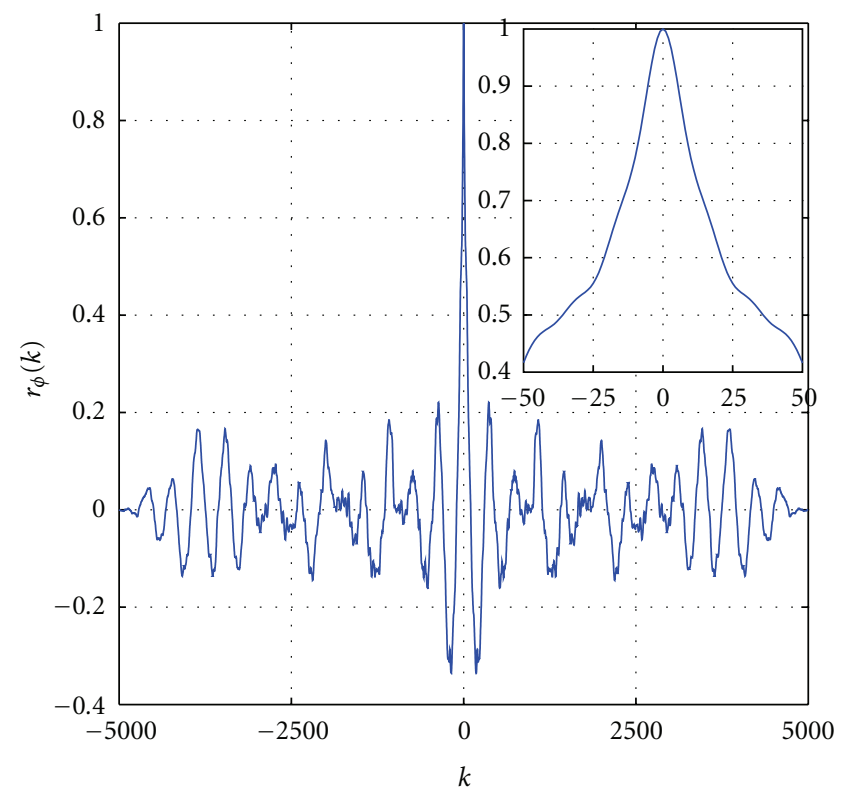

(b) Temporal correlation evaluated over 10000 samples

Figure 4: Temporal phase noise measurements.

$\Delta f^{(3)}=15.45 \mathrm{kHz}$, and $\Delta f^{(4)}=15.46 \mathrm{kHz}$. The standard deviation is approximately $100 \mathrm{~Hz}$. Similarly to the DC offset case, to study the statistics of the carrier frequency offsets we would need a wide set of receivers, which is not doable in our case. However, a Gaussian distribution can be inferred. Furthermore, we assume the frequency offset to be independent among the receivers, with identical mean $15 \mathrm{kHz}$ and standard deviation $100 \mathrm{~Hz}$. 


\section{System Model for DOA Estimation}

Using the impairment models derived from measurements, we can obtain an overall system model. In particular, we consider a radio system with a receiver equipped with a $3 \mathrm{D} \mathrm{L-}$ shaped antenna array [19] as shown in Figure 5. It has a total of $3 M-2$ sensors with three linearly equispaced subarrays displaced along the $x, y$, and $z$ axes. Assuming a source that emits a single tone signal $e^{j 2 \pi f_{0, \mathrm{RF}} t}$ at radio frequency (RF) $f_{0, \mathrm{RF}}$, and a plane waves line-of-sight propagation scenario, the incident complex signal at the $i$ th sensor of the ath antenna subarray can be written as

$$
x_{\mathrm{RF}}^{(a, i)}(t)=\rho e^{\mathrm{j} 2 \pi f_{0, \mathrm{RF}}\left(t-\tau^{(a)}-\Delta t^{(a, i)}\right)}+w_{\mathrm{RF}}^{(a, i)}(t),
$$

where $\tau^{(a)}$ is the propagation delay between the emitter and the first sensor of each subarray, $\rho$ is the propagation loss that we assume to be time invariant during the DoA estimation and identical for each antenna element, and $w_{\mathrm{RF}}^{(a, i)}(t)$ is the additive noise. It should be noted that the index $i$ belongs to $\{1, \ldots, M\}$, while the index $a$ belongs to $\{x, y, z\}$. Now, let us assume that the plane wave impinges on the sensors of every subarray with azimuth $\phi$ and elevation angle $\vartheta$. Then, the differential propagation delay between the first sensor and the $i$ th sensor of a given subarray $a$ can be written as

$$
\Delta t^{(a, i)}= \begin{cases}\frac{d}{c_{0}}(i-1) \cos (\phi) \sin (\vartheta), & a=x, \\ \frac{d}{c_{0}}(M-i) \sin (\phi) \sin (\vartheta), & a=y, \\ \frac{d}{c_{0}}(i-1) \cos (\vartheta), & a=z,\end{cases}
$$

where $d$ is the distance between the sensors, $c_{0}$ is the speed of light, and we assume that $\phi \in[-\pi, \pi]$ and $\vartheta \in[0, \pi]$.

The received RF signals are downconverted to low frequency $f_{0}$ using a direct-conversion receiver architecture as depicted in Figure 1 for the sensor of indices $(a, i)$. The signal downconversion for each sensor is obtained with a local oscillator with frequency $f_{\mathrm{LO}}^{(a, i)}=f_{0, \mathrm{RF}}-f_{0}-\Delta f^{(a, i)}$, where $\Delta f^{(a, i)}$ represents the carrier frequency offset. The desired low frequency $f_{0}$ is chosen larger than 0 to filter out the DC offset component at zero frequency as discussed here in after.

Now, assuming to sample the signals with period $T$, the sequence of complex samples $x^{(a, i)}(n T)$, associated to the $i$ th sensor of the ath subarray, can be written according to the results in Section 2 as

$$
x^{(a, i)}(n T)=S e^{j\left(\psi^{(a, i)}(n T)+\varphi^{(a, i)}(n T)-\Phi^{(a, i)}\right)}+w^{(a, i)}(n T)+w_{\mathrm{DC}}^{(a, i)},
$$

where

$\psi^{(a, i)}(n T)=2 \pi\left(f_{0}+\Delta f^{(a, i)}\right) n T-2 \pi f_{0, \mathrm{RF}} \tau^{(a)}-2 \pi f_{0, \mathrm{RF}} \Delta t^{(a, i)}$.

$\varphi^{(a, i)}(n T)$ represents the phase noise process and $\Phi^{(a, i)}$ is the phase offset for the sensor of indices $(a, i)$. Further-

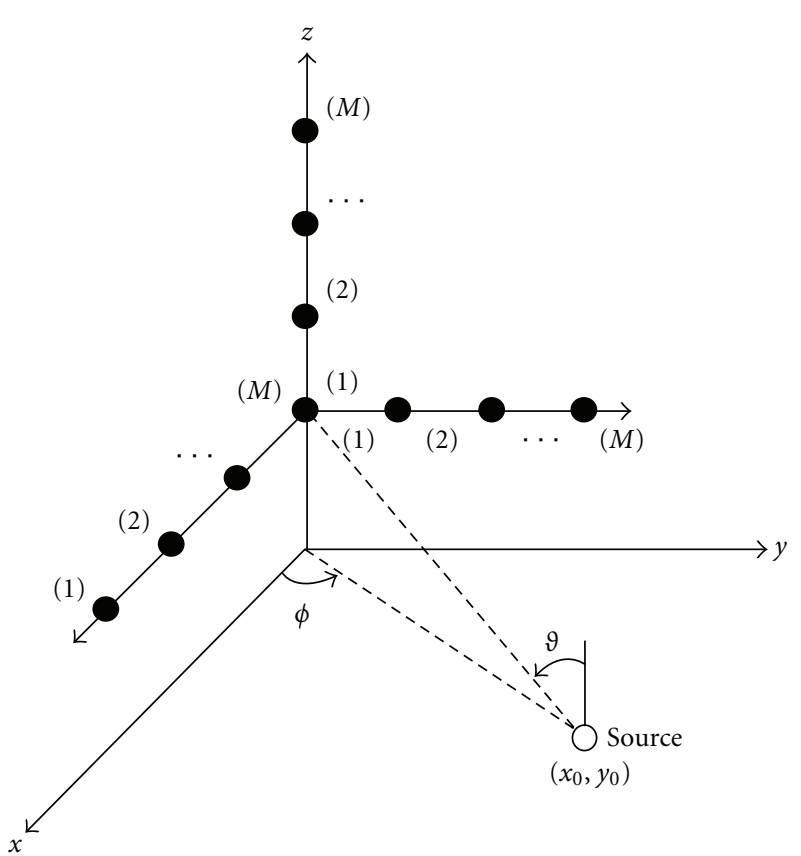

FIGURE 5: 3D L-shaped array configuration.

more, $S=\rho g$ is the signal amplitude (assumed to be identical for each receiver) that includes the propagation loss $\rho$ and the receiver gain $g$. We denote by $w^{(a, i)}(n T)$ the background noise contribution that is white circularly symmetric complex Gaussian, with zero mean, variance $N_{0} / 2$ per component. Finally, $w_{\mathrm{DC}}^{(a, i)}$ is the constant DC offset component.

For the performance analysis, it is useful to define the signal-to-background noise ratio (SNR) as

$$
\mathrm{SNR}=\frac{S^{2}}{N_{0}} .
$$

Our goal is the estimation of the DoA, that is, the estimation of the azimuth and elevation angles, by observing the signals (6). This can be done through the exploitation of the phase differences $2 \pi f_{0, \mathrm{RF}} \Delta t^{(a, i)}$ among the sensors that are due to different propagation delays of signals captured by the sensors. Unfortunately, the presence of hardware impairments, in particular the carrier frequency offset $\Delta f^{(a, i)}$, the phase offset $\Phi^{(a, i)}$, and the phase noise $\varphi^{(a, i)}(n T)$ causes a phase uncertainty as shown in (6) and (7). Furthermore, we have to deal with the DC offset component $w_{\mathrm{DC}}^{(a, i)}$. To compensate these hardware impairments we propose the algorithm described in the next section.

\section{Impairment Compensation and 2D DoA Estimation}

The proposed algorithm for the estimation of the DoA in the presence of the hardware impairments comprises the following main steps.

(i) We filter the signals to mitigate the DC offset and we perform a precalibration procedure to estimate the 
phase offsets among all the receiving antennas and compensate them.

(ii) We correlate pairs of antenna element signals to remove the carrier frequency offsets. In the following we refer to the correlation of signals, that is, the product of a signal with the complex conjugate of another, as differential operation.

(iii) We perform averaging in time and space and finally compute the angles of arrival $(\phi, \theta)$.

We now discuss in more detail the algorithm.

4.1. DC Offset Compensation. To attenuate the DC offset we filter the acquired signal $x^{(a, i)}(n T)$ with a high-pass filter choosing a $f_{0}$ larger than 0 . In this way we obtain SDR values larger than those in Figure 2. In the remainder of this paper, wherever DC offset is mentioned, it means the uncompensated part of the DC offset due to the limited filter attenuation.

4.2. Frequency and Phase Offset Compensation. We assume the carrier frequency offsets to be identical on the same subarray, that is, $\Delta f^{(a, i)}=\Delta f^{(a)}$, for all $i \in\{1, \ldots, M\}$. This assumption is practically true from the results in Section 2. Nevertheless, the effect of nonidentical carrier frequency offsets as well as the effect of the phase noise (that we neglect in the algorithm derivation) will be considered later. Then, we perform a differential operation among pairs of signals from elements that belong to the same subarray. Now, the signal at the output of the differential combiner can be written as

$$
\begin{aligned}
z^{(a, i)}(n T) & =x^{(a, i)}(n T) \cdot x^{(a, i+1)^{*}}(n T) \\
& =A e^{j\left(\hat{\psi}^{(a)}-\widehat{\Phi}^{(a, i)}\right)}+\widehat{w}^{(a, i)}(n T), \quad i \in\{1, \ldots, M-1\},
\end{aligned}
$$

where $A=S^{2}$, and

$$
\widehat{\psi}^{(a)}= \begin{cases}2 \pi f_{0, \mathrm{RF}} \frac{d}{c_{0}} \cos (\phi) \sin (\vartheta), & a=x \\ -2 \pi f_{0, \mathrm{RF}} \frac{d}{c_{0}} \sin (\phi) \sin (\vartheta), & a=y, \\ 2 \pi f_{0, \mathrm{RF}} \frac{d}{c_{0}} \cos (\vartheta), & a=z .\end{cases}
$$

As (9) reveals, the time variant phase ambiguity $2 \pi\left(f_{0}+\right.$ $\left.\Delta f^{(a)}\right) n T$ introduced by the carrier frequency offset in (7) is removed, and we are left with (a) the phase $\widehat{\psi}^{(a)}$ of interest that is a function of the angles of arrival $(\phi, \theta)$, according to (10) and (b) the phase difference $\widehat{\Phi}^{(a, i)}=\Phi^{(a, i)}-\Phi^{(a, i+1)}$, $a \in\{x, y, z\}, i \in\{1, \ldots, M-1\}$ between the $i$ th and the $(i+1)$ th receiver of the ath subarray.

Now, we consider the phase offset $\widehat{\Phi}^{(a, i)}$ compensation. In our test bed, we have carefully designed the antenna array so that the contribution to $\Phi^{(a, i)}$ is due to both not cophased local oscillators and fabrication variations in the $\mathrm{RF}$ receiver stage. We generate a local signal that is used to compensate $\hat{\Phi}^{(a, i)}$ by injecting it to each input receiver. In this way, the phases $\hat{\psi}^{(a)}, a \in\{x, y, z\}$ in (9) become null, and we can estimate the phase offset $\widehat{\Phi}^{(a, i)}$ from (9). Hence, we assume to perform the precalibration just before the $2 \mathrm{D}$ DoA estimation, that is, to time multiplex the acquisition of the reference signal and the acquisition of the unknown direction signals.

4.3. 2D DoA Estimation. The DoA can be estimated from (9). The samples $z^{(a, i)}(n T)$ in (9) are averaged in time and in space to increase the immunity to noise after the compensation of the phase offset $\widehat{\Phi}^{(a, i)}$ (estimated in the precalibration procedure) as follows:

$$
\begin{aligned}
z^{(a)} & =\frac{1}{N(M-1)} \sum_{n=0}^{N-1} \sum_{i=1}^{M-1} z^{(a, i)}(n T) e^{j \hat{\Phi}^{(a, i)}} \\
& =A e^{j \hat{\psi}^{(a)}}+W^{(a)}
\end{aligned}
$$

where $W^{(a)}$ is the complex noise contribution.

Now, with the array configuration in Figure 5 we can process the signals $z^{(x)}, z^{(y)}$, and $z^{(z)}$ in (11) to estimate the angles of arrival as follows:

$$
\begin{gathered}
\tilde{\phi}=-\arctan 2\left(\angle z^{(y)}, \angle z^{(x)}\right), \\
\widetilde{\vartheta}=\arctan 2\left(\sqrt{\left(\angle z^{(x)}\right)^{2}+\left(\angle z^{(y)}\right)^{2}}, \angle z^{(z)}\right),
\end{gathered}
$$

where $\arctan 2(y, x)$ is defined as

$$
\arctan 2(y, x)= \begin{cases}\arctan \left(\frac{y}{x}\right), & x>0, \\ \pi+\arctan \left(\frac{y}{x}\right), & y \geq 0, x<0, \\ -\pi+\arctan \left(\frac{y}{x}\right), & y<0, x<0, \\ \frac{\pi}{2}, & y>0, x=0, \\ -\frac{\pi}{2}, & y<0, x=0 .\end{cases}
$$

\section{Performance Analysis}

In this section we report the performance analysis of the proposed algorithm in terms of aggregate root mean-squared error (RMSE) defined as

$$
\operatorname{RMSE}=\sqrt{E\left\{(\phi-\tilde{\phi})^{2}+(\vartheta-\tilde{\vartheta})^{2}\right\}},
$$

where $E\{\cdot\}$ is the expectation operator.

In the numerical examples, we have assumed $1 / T=$ $50 \mathrm{MHz}, f_{0, \mathrm{RF}}=2.415 \mathrm{GHz}, f_{0}=1 \mathrm{MHz}$, and antenna elements spaced by $d=\lambda_{0} / 2$. Also, where not specified, we have considered $N=1$. The number of elements of the array is $3 M-2=10$; in particular $M=4$ antennas per subarray 
are used with the central element shared among the three subarrays. We have found, via simulation, that with this number of antennas we obtain good performance in the DoA estimation. It should be noted that the required number of channels is larger than that of the hardware configuration that we have used for characterization of the impairments. However, the model we have derived is applicable to any number of antennas. The carrier frequency offsets have been assumed constant over the processing window of duration $N T$ and modeled as spatially independent Gaussian random variables with mean value $m_{\Delta f}=15 \mathrm{kHz}$ and standard deviation $\sigma_{\Delta f}$. The DC offset $w_{\mathrm{DC}}^{(a, i)}$ has been drawn from a complex normal distribution with zero mean and variance $N_{1}$. Finally, the phase noise models have been considered as in Figure 3(a), with a standard deviation of $1^{\circ}$.

In Figure 6, the aggregate RMSE is shown as a function of the azimuth and elevation angles, estimated when SNR = $30 \mathrm{~dB}$ in the absence of impairments. For the sake of graphical representation clarity, the RMSE has been drawn for angular values in the range $\phi=\left[-170^{\circ}, 170^{\circ}\right], \vartheta=$ $\left[10^{\circ}, 170^{\circ}\right]$, and it has been truncated when it exceeds the values of $3^{\circ}$. As we can see, the overall RMSE is always lower than $1.5^{\circ}$, except when $\vartheta=90^{\circ}$ and $\phi=-90^{\circ}, \phi=0^{\circ}$, or $\phi=90^{\circ}$ where the RMSEs have been truncated. In these cases the estimator fails since the argument of the $\arctan 2$ function in (12) goes either to zero or to infinity but it has a wrong sign because of the presence of noise.

Now, we focus our analysis on the effects of the described hardware impairments considering $\phi=30^{\circ}$ and $\vartheta=50^{\circ}$. The results do not significantlly change for different values of $\phi$ and $\vartheta$. To benchmark the performance, we have also considered the root-MUSIC [14] algorithm appropriately adapted to our case. The main steps are described as follows.

5.1. 2D Root-MUSIC. We extend the root-MUSIC algorithm [14] in the following manner. Firstly, we determine the estimated autocorrelation matrix:

$$
\mathbf{R}^{(a)}=\frac{1}{N} \sum_{n=0}^{N-1} \mathbf{x}^{(a)}(n T) \mathbf{x}^{(a)^{H}}(n T),
$$

over $N$ samples for each subarray, where $\mathbf{x}^{(a)}(n T)=$ $\left[x^{(a, 1)}(n T) x^{(a, 2)}(n T) \cdots x^{(a, M)}(n T)\right]^{T}, .^{T}$ is the transposition operator and ${ }^{H}$ is the complex conjugate of the transposition operator. We find the eigendecomposition of $\mathbf{R}^{(a)}=$ $\mathbf{Q}^{(\mathrm{a})} \boldsymbol{\Lambda}^{(\mathrm{a})} \mathbf{Q}^{(a)^{H}}$, and we determine the partition $\mathbf{Q}_{n}^{(a)}$ of the eigenvector matrix $\mathbf{Q}^{(a)}$ associated to the smallest $M-1$ eigenvalues. The $\mathbf{Q}_{n}^{(a)}$ matrix is the eigenvector matrix that spans the noise subspace. From the matrix $\mathbf{C}^{(a)}=\mathbf{Q}_{n}^{(a)} \mathbf{Q}_{n}^{(a)^{H}}$, we obtain the coefficients $C_{l}^{(a)}$ by summing the elements of the $l$ th subdiagonal of $\mathbf{C}^{(a)}$. Now, we compute the $M-1$ roots of the polynomial with coefficients $C_{l}^{(a)}, l \in\{0, \ldots, M-1\}$. Finally, we pick the root closest to the unit circle, namely, $z^{(a)}$. From the values $z^{(a)}, a \in\{x, y, z\}$ we determine the 2D DoA as in (12).

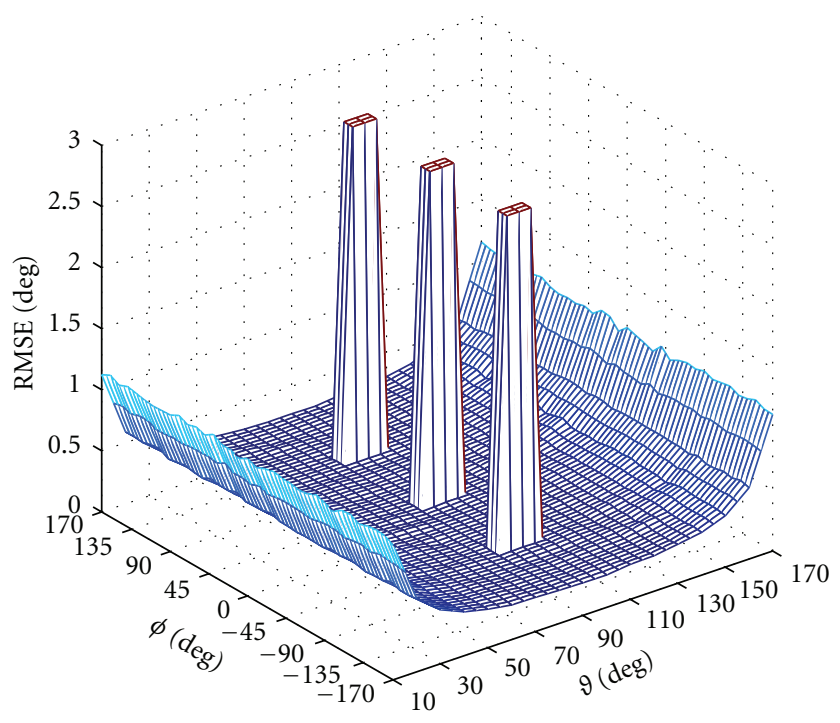

FIGURE 6: Aggregate RMSE as a function of azimuth and elevation angles.

We can observe that the carrier frequency offset is partially compensated with the autocorrelation matrix derivation. In fact, we can interpret it as a sort of extended differential operation among all the possible pairs of antenna elements.

It is clear that the complexity of this algorithm is considerably higher than the proposed one.

5.2. Hardware Impairment Effects and Comparison with RootMUSIC. In Figure 7(a), we can observe the aggregate RMSE as a function of SNR for some different values of SDR measured at the output of the DC offset compensationstage.

As we can see an error floor in the RMSE curves is determined by the presence of the DC offset. The larger the DC offset power (the lower the SDR), the larger the RMSE is.

A comparison between the algorithm in Section 4 and the root-MUSIC is shown in Figure 7(b). This has been done for two different values of DC offset power, SDR $=30 \mathrm{~dB}$ and $\mathrm{SDR}=50 \mathrm{~dB}$. The proposed estimator exhibits better performance than the root-MUSIC and it provides lower error floors.

In Figure 8(a), we consider the case of unequal frequency offsets among the channels. The aggregate RMSE curves are shown as a function of the carrier frequency offset standard deviation $\sigma_{\Delta f}$ and the number of snapshots $N$, with $\mathrm{SNR}=30 \mathrm{~dB}$. We have not considered the carrier frequency offset mean value $m_{\Delta f}$ as a simulation parameter because it is perfectly compensated by the algorithm; so it is not a source of error. We can observe that the larger $\sigma_{\Delta f}$, is the larger the RMSE is. Furthermore, for low values of $\sigma_{\Delta f}$, the increase of $N$ is beneficial because the average reduces the noise. But, with the increase of $\sigma_{\Delta f}$, the increase of $N$ is detrimental because the phase error introduced by the nonperfect compensation of the carrier frequency offset is 


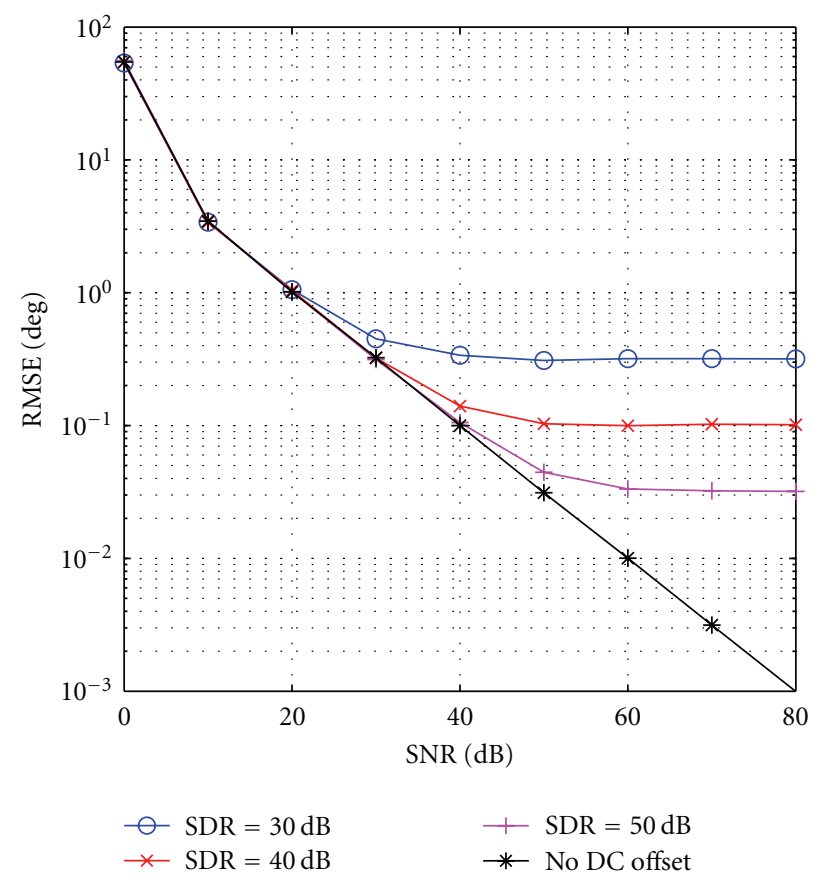

(a) Our estimator

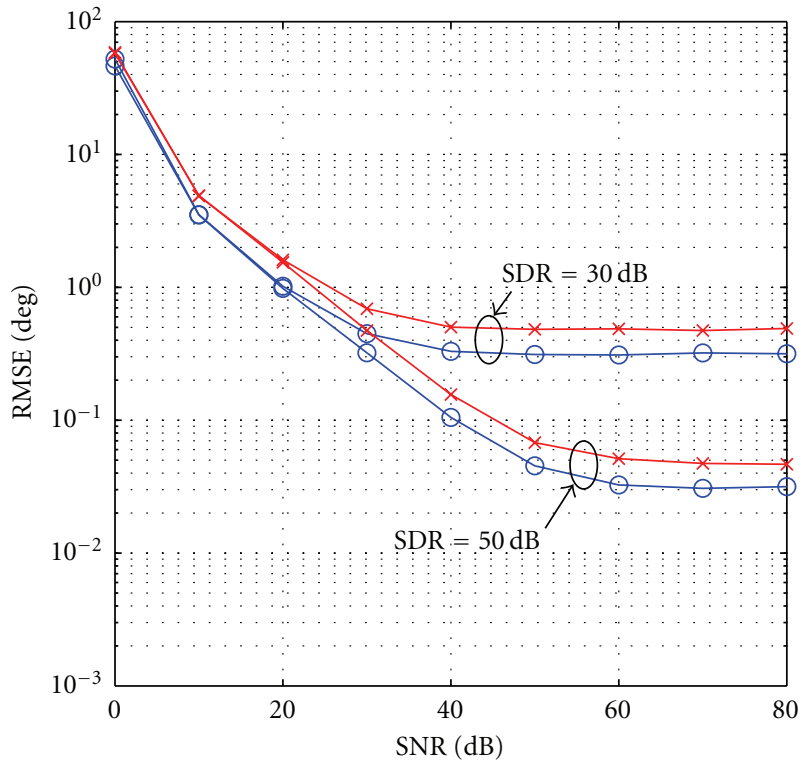

$\ominus$ Our estimator

$*$ Root-MUSIC

(b) Comparison with root-MUSIC

FIgURE 7: Aggregate RMSE as a function of SNR and SDR.

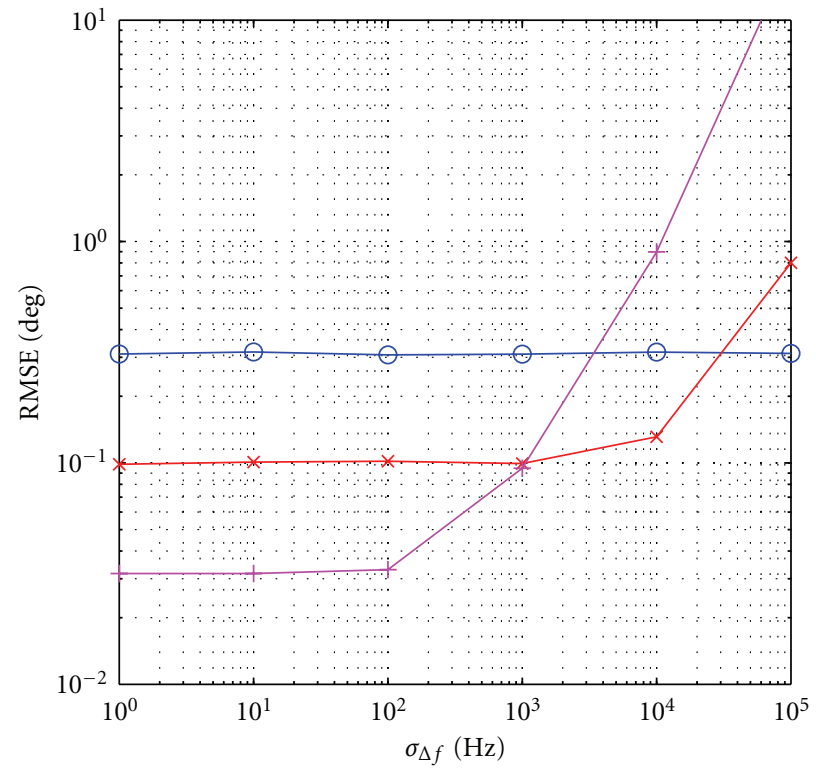

$\bigcirc N=1$

$\rightarrow N=10$

$+N=100$

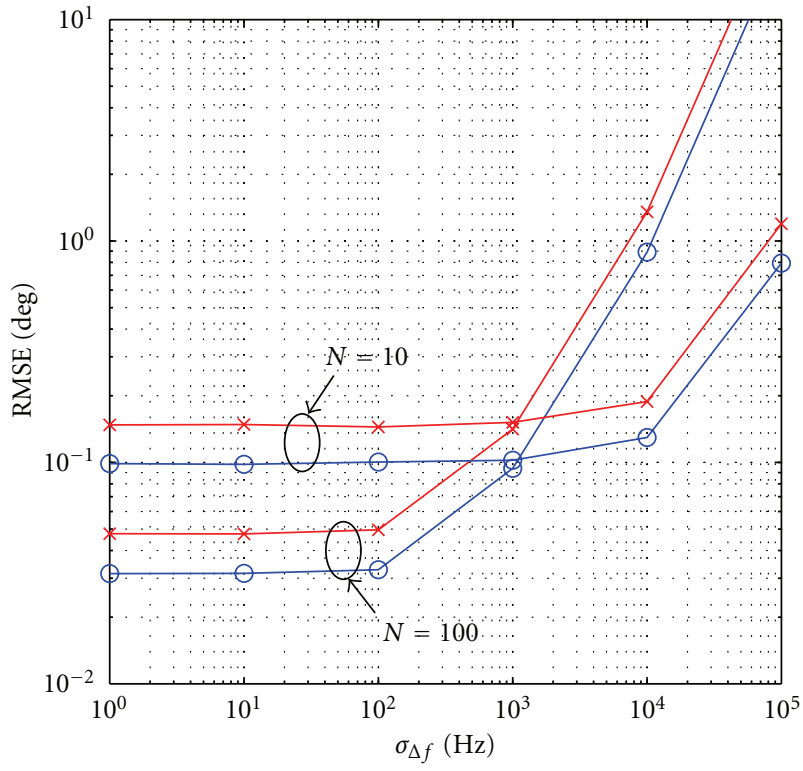

$\bigcirc$ Our estimator

$*$ Root-MUSIC

(b) Comparison with root-MUSIC

(a) Our estimator

FIgURE 8: Aggregate RMSE as a function of $\sigma_{\Delta f}$ and $N$. 


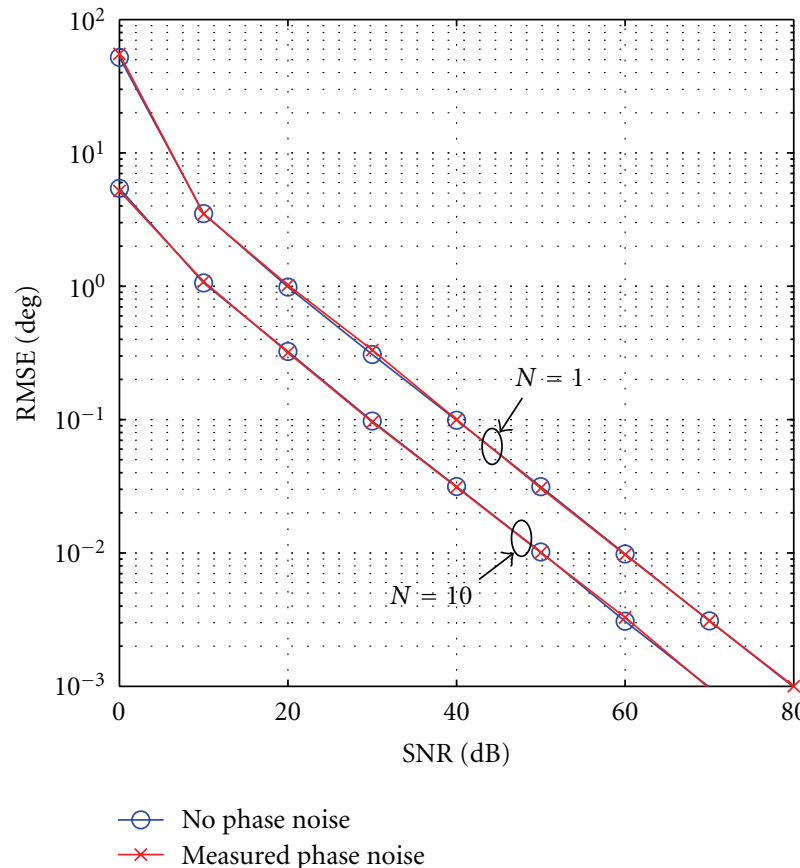

(a) Our estimator

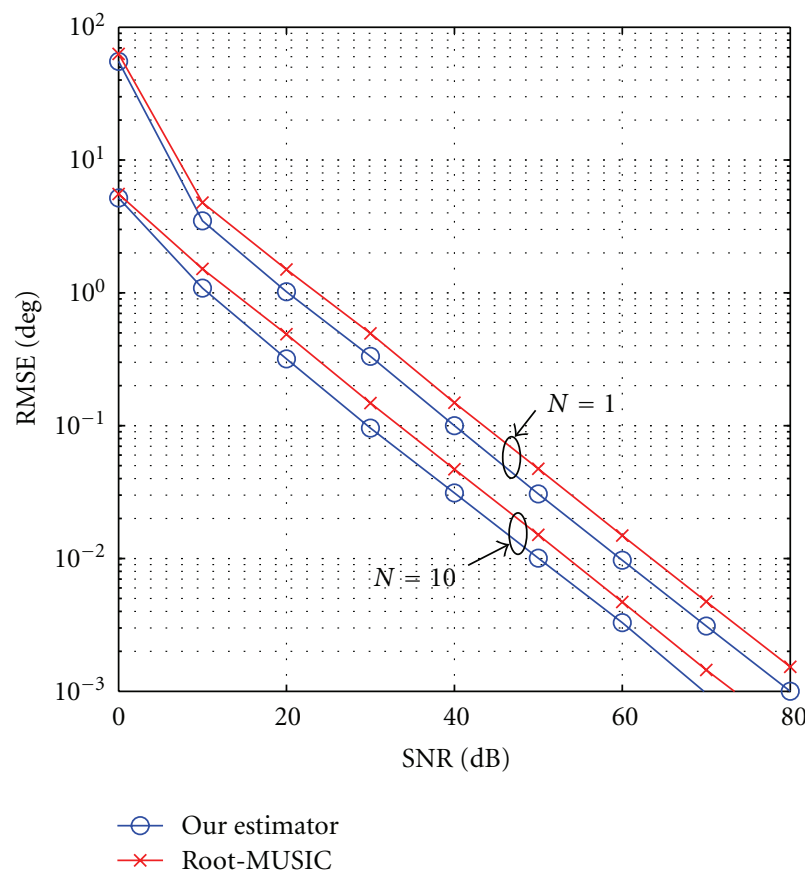

(b) Comparison with root-MUSIC in the presence of phase noise

FIGURE 9: Aggregate RMSE as a function of SNR, $N$, and the presence or not of phase noise.

accumulated over $N$ samples. In fact, if $\Delta f^{(a, i)} \neq \Delta f^{(a, i+1)}$, $a \in\{x, y, z\}, i \in\{1, \ldots, M-1\},(11)$ becomes

$$
z^{(a)}=\eta^{(a)} A e^{j\left(\psi^{(a)}+\epsilon^{(a)}\right)}+W^{(a)},
$$

where

$$
\eta^{(a)} e^{j \epsilon^{(a)}}=\frac{1}{N(M-1)} \sum_{n=0}^{N-1 M-1} \sum_{i=1}^{j 2 \pi\left(\Delta f^{(a, i)}-\Delta f^{(a, i+1)}\right) n T} .
$$

For this reason, the value $N$ has to be chosen so that a tradeoff is made between the increase of the signal-to-noise ratio and the increase of the phase error due to the carrier frequency offset.

In Figure 8(b), we show a comparison with the rootMUSIC. We have considered the sensitivity to carrier frequency offset for $N=10$ and $N=100$. Even here the rootMUSIC manifests a lower performance.

In Figure 9(a), we have considered the RMSE curves as a function of the SNR, $N$, with or without simulated phase noise. We have considered the phase noise model derived from our test bed in Section 2. Interestingly, we observe that the phase noise does not introduce any significant degradation of performance. This is because the phase noise is partly compensated by the phase offset precalibration procedure. In fact, considering the phase noise contribution, (9) becomes

$$
z^{(a, i)}(n T)=A e^{j\left\{\hat{\psi}^{(a, i)}-\widehat{\Phi}^{(a, i)}-\hat{\varphi}^{(a, i)}(n T)\right\}}+\widehat{w}^{(a, i)}(n T),
$$

where $\hat{\varphi}^{(a, i)}(n T)=\varphi^{(a, i)}(n T)-\varphi^{(a, i+1)}(n T)$ is the resulting phase noise contribution. When the precalibration step is performed, we estimate the total phase as the sum of the contributions of the phase offset $\widehat{\Phi}^{(a, i)}$ and the phase noise $\hat{\varphi}^{(a, i)}(n T)$. Since the phase noise is slowly time variant in the estimation window (see Figure 4(b)), the precalibration procedure that we do just before the 2D DoA estimation compensates well the phase noise contribution.

Figure 9(a) shows that improved performance is obtained with $N=10$.

Finally, in Figure 9(b), we make a comparison with the root-MUSIC. These curves confirm that our estimator performs better than the root-MUSIC, also in the presence of phase noise.

\section{Conclusion}

We have reported several experimental results from measurements done on a test bed to derive a system model for a typical RF direct-conversion hardware architecture affected by DC offsets, carrier frequency offsets, phase offsets, and phase noise. We have presented an algorithm for 2D DoA estimation with 3D L-shaped arrays considering this system model. The proposed estimation algorithm is simple and compensates the carrier frequency offsets via differential signal combining.

The performance of the algorithm has been studied via simulations for several system configurations and parameter setups. Furthermore, a comparison with the well-known root-MUSIC algorithm is performed. The results show that the estimator is robust for a wide range of angles and SNRs, and it performs better than root-MUSIC. Spatial and temporal averaging increases the noise immunity of 
the algorithm. However, while spatial averaging is always beneficial, the temporal averaging window length has to be selected according to the operating SNR in the presence of distinct carrier frequency offsets between the RF receivers of distinct antenna elements. Distinct carrier frequency offsets may or may not exist as a function of the specific RF downconversion architecture.

The effects of DC offset and phase noise are also considered in this work. It is shown that DC offset determines an error floor in the RMSE curves, and the performance of our estimator is better than the one of the root-MUSIC. Finally, it is shown that a phase offset calibration can be done using a local reference signal. It is beneficial to compensate the phase differences of the local oscillators that comprise a contribution due to the slowly time variant phase noise process.

Future work will consist in the implementation of the proposed algorithm in the hardware test bed to perform analysis in the real field.

\section{Acknowledgments}

The authors wish to thank the Editor professor Nikos C. Sagias and the anonymous reviewers for their helpful comments that allowed improving the quality of this manuscript. D. Inserra acknowledges that part of the work herein presented was funded by CNIT, Consorzio Nazionale Interuniversitario per le Telecomunicazioni, with a doctoral research grant.

\section{References}

[1] N. Amitay, R. G. Pecina, and C. P. Wu, "Radiation properties of large planar arrays," Tech. Rep., Bell Telephone Lab, Whippany, NJ, USA, 1965.

[2] A. L. Swindlehurst, "Maximum likelihood doa estimation and detection without eigendecomposition," in Proceedings of the IEEE International Conference on Acoustic, Speech and Signal Processing (ICASSP '92), pp. 401-404, March 1992.

[3] R. O. Schmidt, "Multiple emitter location and signal parameter estimation," IEEE Transactions on Antennas and Propagation, vol. AP-34, no. 3, pp. 276-280, 1986.

[4] R. Roy and T. Kailath, "ESPRIT-estimation of signal parameters via rotational invariance techniques," IEEE Transactions on Acoustics, Speech, and Signal Processing, vol. 37, no. 7, pp. 984-995, 1989.

[5] M. P. Wylie, S. Roy, and H. Messer, "Joint DOA estimation and phase calibration of linear equispaced (LES) arrays," IEEE Transactions on Signal Processing, vol. 42, no. 12, pp. 34493459, 1994.

[6] A. Paulraj and T. Kailath, "Direction of arrival estimation by eigenstructure methods with unknown sensor gain and phase," in Proceedings of the IEEE International Conference on Acoustics, Speech and Signal Processing (ICASSP '88), pp. 26812684, New York, NY, USA, 1988.

[7] C. Wang and J. A. Cadzow, "Direction-finding with sensor gain, phase and location uncertainty," in Proceedings of the International Conference on Acoustics, Speech, and Signal Processing (ICASSP '91), pp. 1429-1432, July 1991.

[8] K. R. Dandekar, H. Ling, and G. Xu, "Smart antenna array calibration procedure including amplitude and phase mismatch and mutual coupling effects," in Proceedings of the
IEEE International Conference on Personal Wireless Communications(ICPWC '00), pp. 293-297, December 2000.

[9] C. Reck, U. Berold, J. Weinzierl, and L. P. Schmidt, "Direction of arrival estimation from secondary surveillance radar signals in presence of hardware imperfections," in Proceedings of the 5th European Radar Conference, pp. 252-255, October 2008.

[10] "Lyrtech website," http://www.lyrtech.com/.

[11] J. G. Proakis, Digital Communications, McGraw-Hill, New York, NY, USA, 3rd edition, 1995.

[12] H. Meyr, M. Moeneclaey, and S. A. Fetchel, Digital Communication Receivers: Synchronization, Channel Estimation, and Signal Processing, Wiley-Interscience, New York, NY, USA, 2nd edition, 1997.

[13] B. Lindoff and P. Malm, "BER performance analysis of a direct conversion receiver," IEEE Transactions on Communications, vol. 50, no. 5, pp. 856-865, 2002.

[14] H. K. Hwang, Z. Aliyazicioglu, M. Grice, and A. Yakovlev, "Direction of arrival estimation using a root-music algorithm," in Proceedings of the International MultiConference of Engineers and Computer Scientists (IMECS '08), March 2008.

[15] D. Inserra and A. M. Tonello, "DoA estimation with compensation of hardware impairments," in Proceedings of the IEEE 72nd Vehicular Technology Conference Fall (VTCFall '10), Ottawa, ON, Canda, September 2010.

[16] D. Inserra, A. M. Tonello, and N. Moret, "Positioning based on 2-d aoa estimation," in Proceedings of the IEEE 73rd Vehicular Technology Conference Spring (VTCSpring '11), Budapest, Hungary, May 2011.

[17] P. B. Kenington, RF and Baseband Techniques for Software Defined Radio, Artech House, Norwood, Mass, USA, 2005.

[18] P. Robertson and S. Kaiser, "Analysis of the effects of phasenoise in orthogonal frequency division multiplex (ofdm) systems," in Proceedings of the 1995 IEEE International Conference on Communications (ICC '95), pp. 1652-1657, Seattle, Wash, USA, June 1995.

[19] Y. Hua, T. K. Sarkar, and D. D. Weiner, "An L-shaped array for estimating 2-D directions of wave arrival," IEEE Transactions on Antennas and Propagation, vol. 39, no. 2, pp. 143-146, 1991. 

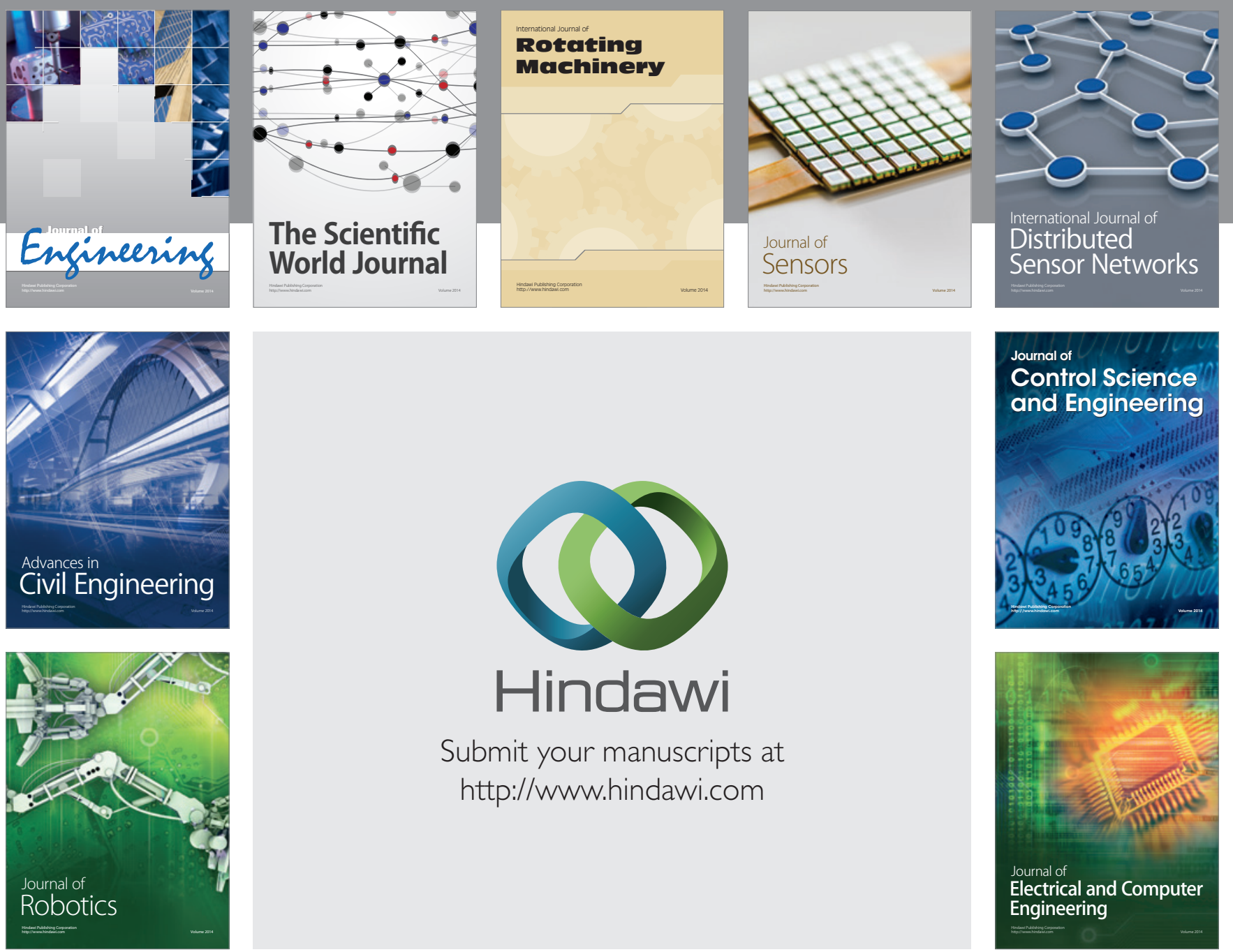

Submit your manuscripts at

http://www.hindawi.com
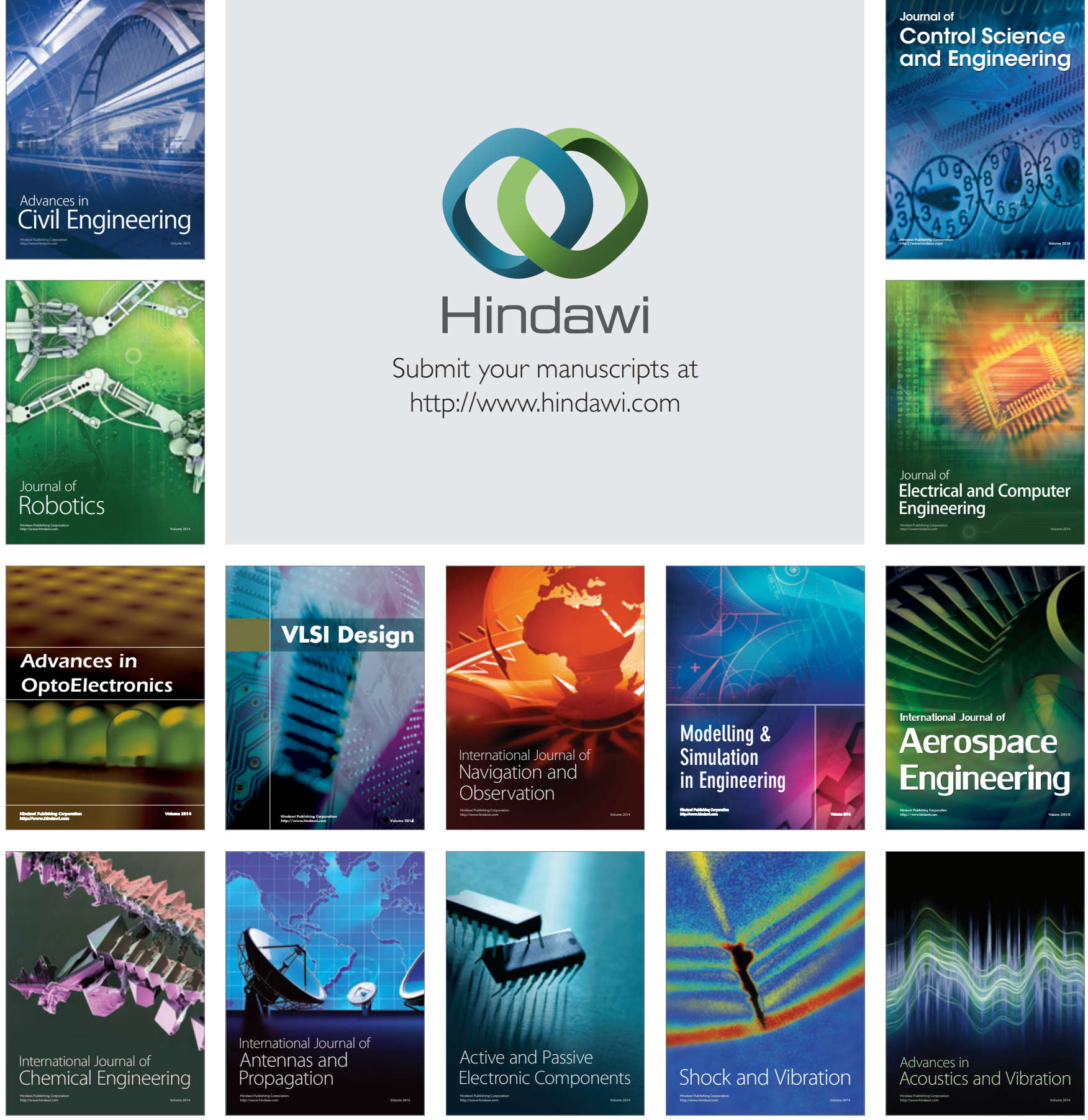